\title{
Autoignition of Nonuniform Mixtures in Chambers of Variable Volume
}

\author{
A. LIÑÁN \\ F. A. WILLIAMS
}

\section{INTRODUCTION}

Combustion in compression-ignition $(\mathrm{CI})$ engines is a complicated process involving turbulent, two-phase flows with transport processes and finite-rate chemistry (Heywood, 1988; Kamimoto and Kobayashi, 1991). Reviews indicate that more accurate descriptions of autoignition in variable-volume chambers are needed for advancing methods of CI-engine design if potential future improvements in Diesel combustion are to be realized. There has been recent progress in both experimental and computational approaches to the investigation of $\mathrm{Cl}$ autoignition (Kamimoto and Kobayashi, 1991). In particular, computational methods have been applied with some success to the calculation of ignition in transient fuel sprays (Aggarwal, 1989; Takagi et al., 1991). These methods, however, suffer from uncertainties concerning how the finite-rate chemistry is treated. The present paper offers an alternative that may help to circumvent these uncertainties.

Computation of nonreacting two-phase, turbulent flows with atomization and evaporation is a challenging problem in itself. The computational uncertainties are greatly increased when the rapid finite-rate chemistry that typically occurs during autoignition is included as well. Therefore, there is motivation for obtaining simplified and potentially more accurate descriptions of the influences of the autoignition chemistry on the overall combustion processes. The present work addresses the problem of obtaining such simplified descriptions. It is presumed here that experiment or numerical simulation has given the spatial distributions of temperature and fuel and oxidizer concentrations within the combustion chamber under inert (nonreacting) 
conditions in the vicinity of the time of initiation of the processes leading to autoignition. An analytical approach based on activation-energy asymptotics (AEA) is then developed to provide the ignition time and subsequent pressure history in terms of this initial input information. The results remove the necessity of including the autoignition chemistry in numerical simulations, enabling them to focus on the transport-controlled stages of evaporation and combustion that occur prior to and subsequent to ignition.

Attention is focused on spontaneous ignition that takes place as a result of the temperature rise that occurs when the chamber volume $V(t)$ is reduced. The ignition transient is assumed to be short compared with characteristic times of heat conduction across nonhomogeneities, so that heat-conduction and diffusion effects can be neglected during ignition. In addition, the ignition time is taken to be long compared with the time required for acoustic waves to propagate across the chamber, so that the chamber pressure $P(t)$ can be considered to be spatially uniform, a function only of time $t$. The activation energy of the model one-step reaction is presumed to be large enough that changes in reactant concentrations can be neglected in evaluating reaction rates during ignition. Finally, subsequent to ignition at any point, the ignition front and its associated heat release are considered to propagate more rapidly than the velocity of a premixed laminar flame, measured in the product gas at the adiabatic flame temperature behind the ignition front, under which condition ignition-front propagation dominates any flame propagation that involves transport processes (Zel'dovich, 1980; Dold, 1985, 1988). This last assumption is supported by observed propagation rates up to $60 \mathrm{~m} / \mathrm{s}$ (IDEA, 1993), a value much too high to involve either laminar or turbulent transport.

The idea of the sequence of stages that prevails is illustrated schematically in Figure 1. The turbulent transport processes of evaporation and mixing start during the

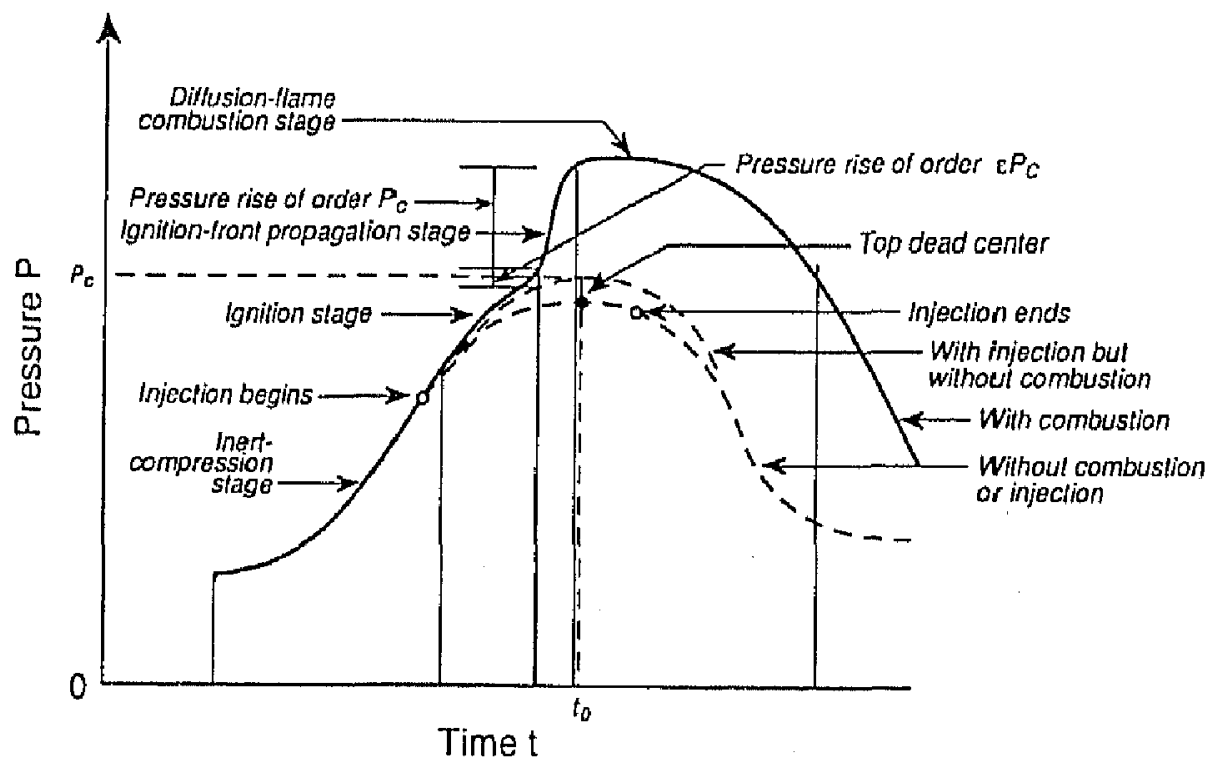

FIGURE 1 Schematic diagram of pressure-time history, illustrating stages introduced here; vertical lines mark boundaries of stages. 
inert compression stage when fuel injection begins. To avoid detrimental effects of early and strong ignition, injection typically begins within thirty crank-angle (CA) degrees of top dead center (TDC), and extends through the ignition stage. Nonreacting-flow computations apply during the inert stage and also in the nonreacting portion of the gas (which occupies most of the chamber) during the ignition stage. The major sharp combustion-induced pressure rise is considered to occur during the ignition-front propagation stage which follows the ignition stage. This stage is considered too short to allow significant mixing and is assumed to be followed directly by a diffusion-controlled combustion stage in which diffusion flames resulting from the nonuniform mixture-fraction field further support pressure increases over a longer time scale. In the fast-chemistry diffusion-controlled limit of large Damköhler numbers, this stage can also be described computationally through turbulent-mixing approaches that do not involve finite-rate chemistry. Since premixed-flame propagation (involving transport) seems likely to be relatively weak and unimportant in contributing to pressure rises in most applications, no such stage is shown between the stages of ignition-front propagation and diffusionflame combustion in Figure 1. Thus, these approximations enable the entire history to be addressed through numerical computations that do not involve finite-rate chemistry.

According to the present viewpoint, the role of vaporization in the autoignition process is only to supply gaseous fuel in a transport-controlled manner. Phase equilibrium is assumed (quite reasonably) to exist for all times at liquid-gas surfaces. With injection extending from before ignition to after ignition-front propagation, vaporization is proceeding throughout the stages analyzed here. However, since the vaporization is transport-controlled, like mixing it occurs to a negligible extent during the short stage of ignition-front propagation. Vaporization must be taken into account in the analyses that describe the stages of inert compression and of diffusion-controlled combustion, and this contributes to the complexity of the transport-controlled spray descriptions required. Nevertheless, since the evaporation processes are an integral part of the injection and mixing, they need no special treatment in the analyses presented here.

Although real, detailed ignition chemistry is more complicated than the one-step AEA description adopted in the present approach, Müller, Peters and Liñán (1992) have recently shown that an empirical four-step mechanism is capable of describing quite well the results of more complex descriptions. For hydrocarbon fuels there are low-temperature and high-temperature ignition regimes having fairly strong positive sensitivity of overall ignition rates to temperature, separated by an intermediatetemperature regime (about $900 \mathrm{~K}$ to $1100 \mathrm{~K}$ ) that exhibits a mildly negative temperature sensitivity. Under any given set of specific conditions away from the regime of negative temperature dependence, it is possible to develop a one-step approximation, of the type employed here, that yields agreement with the predictions of the four-step mechanism (see, however, Appendix A, which addresses this question in somewhat greater detail). Therefore, the influences of real chemistry can generally be incorporated within the framework of the present analysis. It is necessary only to identify the effective overall one-step rate parameters. Values of such parameters may be found, for example, in Heywood (1988, p. 544). 


\section{FORMULATION}

Since molecular diffusion is absent during the periods addressed, it is convenient to employ a Lagrangian description in which $\mathbf{x}$ is the initial position of the fluid particle. Here "initial" means a time in the vicinity of the ignition stage in Figure 1 and typically corresponds to inert conditions near TDC. The initial distribution of the fuel mass fraction $Y_{F}$, the oxidizer mass fraction $Y_{O}$ and temperature $T$ are specified as $Y_{F_{0}}(\mathrm{x})$, $Y_{O_{o}}(\mathbf{x})$ and $T_{o}(\mathbf{x})$, respectively with $Y_{O_{v}}=Y_{O_{x}}\left(1-Y_{F_{o}}\right)$ as a consequence of the nonreactive mixing if the small difference between oxygen and nitrogen diffusivities is neglected. Both deterministic and probabilistic descriptions of the initial spatial distributions will be permitted; in the latter case, for the following development these functions are to be considered to correspond to a particular realization.

Let $D / D t$ denote the material derivative following a fluid element. Then the equations for species and energy conservation become

$$
\begin{aligned}
& D Y_{F} / D t=-B Y_{F}^{n} Y_{O}^{m} e^{-E / R^{0} T}, \\
& D Y_{o} / D t=-v_{O} B Y_{F}^{n} Y_{O}^{m} e^{-E / R^{a} T}
\end{aligned}
$$

and

$$
\rho c_{p} D T / D t=d P / d t+\rho q B Y_{F}^{n} Y_{o}^{m} e^{-E / R^{0} T},
$$

where the reaction orders are $n$ and $m, E$ and $R^{0}$ denote the activation energy and the universal gas constant, $B$ is the effective frequency factor for fuel consumption and $v_{o}$ the stoichiometric oxidizer-to-fuel mass ratio, $\rho$ represents density and $c_{p}$ the specific heat at constant pressure, and $q$ is the heat release per unit mass of fuel. In the gas phase, the ideal-gas equation of state is adopted,

$$
P=\rho\left(R^{0} / W\right) T,
$$

where $W$ represents the average molecular weight, and this enables Equation (3) to be rewritten as

$$
D(\ln T) / D t=[(\gamma-1) / \gamma] d(\ln P) d t+\left[q /\left(c_{p} T\right)\right] B Y_{F}^{n} Y_{o}^{m} e^{-E / R^{\alpha} T},
$$

where the ratio of specific heats,

$$
\gamma=\left(c_{p}-R^{0} / W\right) / c_{p},
$$

will be taken to be constant.

Subtraction of $v_{o}^{-1}$ times Equation (2) from Equation (1) removes the chemical term and enables integration to be performed to show that each fluid element maintains a constant value of mixture fraction, defined as

$$
Z(\mathbf{x})=\left[Y_{F}-\left(Y_{O}-Y_{O_{\infty}}\right) / v_{o}\right] /\left(1+Y_{O_{x}} / v_{o}\right),
$$

where $Y_{O_{x}}$ is the oxidizer mass fraction in the inlet oxidizer stream, and the inlet fuel stream has been assumed to be pure fuel, as is usually true. This relationship enables the concentration factor in the reaction rate to be written in terms of $Y_{F}$ as

$$
Y \equiv Y_{F}^{n} Y_{O}^{m}=Y_{F}^{n}\left[Y_{O_{\infty}}+v_{O} Y_{F}-\left(v_{O}+Y_{O_{\infty}}\right) Z\right]^{m},
$$


so that Equation (1) becomes

$$
d Y_{F} / d t=-B Y e^{-E /\left(R^{\circ} T\right)}
$$

It is convenient to write Equation (5) in terms of an entropy-related variable as

$$
\left.D(\ln S) / D t=\left\{q /\left[c_{p} S P^{(\gamma-1) / \gamma}\right]\right\} B Y e^{-E /\left[R^{v} S \rho^{\alpha_{i}-1 / y_{i}}\right]}\right],
$$

where

$$
S=T / P^{(\gamma-1) / \gamma}
$$

In working with $S$, the exponential of the entropy divided by $c_{p}$, the isentropic increase in $T$ with increasing $P$ during adiabatic compression comes automatically rom Equation (11) with $S$ constant, and the additional compression term is not present in Equation (10). Equations (9) and (10) are the differential equations that, when complemented by the pressure-volume relationship given below, describe the autoignition process addressed here. They are to be integrated subject to the initial distributions at $t=t_{0}$ specified earlier.

\section{PRESSURE-VOLUME RELATIONS}

If $d V_{o}$ is the volume of a fluid element at time $t_{o}$ and $d V$ the volume of the same element (of the same mass) at time $t$, then $\rho d V=\rho_{a} d V_{a}$, where $\rho_{a}(\mathbf{x})$ is the initial density. This relationship implies that the chamber volume $V$ at time $t$ is

$$
V=\iint_{V_{o}} \int\left(\rho_{o} / \rho\right) d V_{o}
$$

Under the assumption that the volume occupied by liquid is negligible compared with that occupied by gas, Equations (4) and (11) may be used in this expression to show that

$$
\left(P / P_{o}\right)^{1 / \gamma}=\left(V_{o} / V\right) \iint_{V_{o}} \int\left(S / S_{o}\right)\left(W_{o} / W\right) d V_{o} / V_{o} .
$$

Prior to chemistry occurring, $S=S_{0}$ and $W=W_{0}$, so that Equation (13) provides the usual isentropic relationship. During this inert stage, a volume decrease produces a pressure increase and a temperature increase.

The time dependence of the chamber volume in the vicinity of TDC can be approximated well by the formula

$$
V=V_{o}\left[1+\left(t-t_{o}\right)^{2} / t_{c}^{2}\right]
$$

where $V_{o}, t_{o}$ and $t_{c}$ are constants. Here $t_{o}$ is the time at TDC, and $t_{c}$ is a characteristic time scale for variations of $V$, obtained from an expansion for $V$ about $t=\mathrm{t}_{o}$ that remains reasonably accurate within about $30^{\circ} \mathrm{CA}$ on each side of TDC. Since the phenomena addressed here occur within this range in the applications, Equation (14) will be employed in the present study. The "initial" conditions at time $t_{o}$ therefore henceforth are inert conditions at TDC, which may be obtained computationally from descriptions of injection, atomization, evaporation and turbulent mixing, extended to 
TDC with chemistry suppressed. Advantages of this selection are that descriptions of ignitions at times corresponding to $\left(t-t_{0}\right) / t_{c}$ of order unity both before and after TDC can readily be recovered from the results, and a criticality for ignition is exhibited which cannot be derived easily from other selections.

\section{THE IGNITION STAGE}

The small parameter of expansion for the ignition stage is

$$
\varepsilon=R^{0} T_{c} / E
$$

where $T_{c}$ is the maximum inert temperature anywhere in the chamber at $t=t_{b}$. Since the vaporized fuel cools the air, the temperature may be expected to reach $T_{c}$ in air away from the walls and not yet mixed with fuel, and very little cycle-to-cycle variation in $T_{c}$ should be anticipated, so it is treated as a known constant in the analysis. According to Equation (11), if $S_{c}$ denotes the maximum value of $S_{o}(\mathbf{x})$ within the chamber and $P_{c}$ the pressure in the inert stage at time $t_{0}$, then

$$
T_{c}=S_{c} P_{c}^{(\gamma-1) / \gamma}
$$

A distinguished limit is that in which the inert solution $S_{0}(\mathbf{x})$ differs from $S_{c}$ by an amount of order 8 throughout the chamber,

$$
S_{o}(\mathbf{x}) / S_{c}=1-\varepsilon S_{0}(\mathbf{x}),
$$

with $S_{0}(\mathbf{x})$ nonnegative and of order unity. Typically, variations in $S_{0}(\mathbf{x})$ will be greater than this, so that $s_{o}(\mathbf{x})$ is large in much of the chamber and is of order unity only in a fraction of the initial volume $V_{o}$. In the latter case, the analysis of the ignition stage automatically receives no contribution from the fraction of the chamber in which $s_{a}(\mathbf{x})$ is large, and therefore no modification of the analysis is needed.

The expansions

$$
\left(t-t_{o}\right) / t_{c}=\varepsilon^{1 / 2} \tau, \quad P / P_{c}=1+\varepsilon p, \quad V / V_{o}=1+\varepsilon \tau^{2}, \quad S / S_{c}=1+\varepsilon\left(s-s_{o}\right)
$$

are introduced, where $p$ is a function of $\tau$, while $s$ depends on $\tau$ and $\mathbf{x}$; these functions are of order unity when $\tau$ is of order unity. The value of $\varepsilon$ is presumed to be small enough that reactant consumption (and therefore changes in $Y$ and $W$ ) can be neglected during ignition. Therefore, only Equation (10) needs to be considered. The expansion of Equation(10) to leading order in $\varepsilon$ is

$$
D s / D \tau=\delta Y_{0} e^{s-s_{0}+[(\gamma-1) / \gamma] p,}
$$

where the Frank-Kamenetskii parameter is

$$
\delta=\frac{t_{c} q B_{c}}{c_{p} T_{c}} e^{-E / R^{0} T_{c}} \varepsilon^{-1 / 2},
$$

in which $B_{c}$ denotes the value of $B$ at pressure $P_{c}$, and in general $q B_{c} / c_{p}$ may vary with $\mathbf{x}$, so that $\delta$ varies, but typically not appreciably, and for simplicity here it is therefore treated as a known constant. It may be noted that $\delta Y_{o}$ is the ratio of the characteristic 
compression time $t_{c} \varepsilon^{1 / 2}$, required to change the volume around $V_{o}$ by an amount $\varepsilon V_{b}$, to the adiabatic, constant-pressure ignition time. The expansion of Equation (13) shows that

$$
p=\gamma\left(\bar{s}-\tau^{2}\right),
$$

which may be used in Equation (19) to express $p$ in terms of $\tau$ and the volume average of $s$, denoted by an overbar, namely,

$$
\bar{s}=\iint_{V_{a}} \int s d V_{v} / V_{a}
$$

A similar volume integral was introduced by Kassoy et al. (1989) who considered ignition of a homogeneous mixture of nonuniform temperature in a constant-volume chamber. In general, Equation (9) becomes

$$
D Y_{F} / D \tau=-\left(c_{p} T_{c} / q\right) \varepsilon \delta Y e^{s-s_{o}+[(\gamma-1) / \gamma] p},
$$

which suggests that reactant consumption can be neglected to leading order because of the factor $\varepsilon$, not present in Equation (19).

Equations (19) and (21) show that the equation to be integrated is

$$
D S / D \tau=\left(\delta Y_{o} e^{-s_{a}}\right) e^{-(\gamma-1) \tau^{2}+(\gamma-1) s} e^{s},
$$

subject to the matching condition $s \rightarrow 0$ as $\tau \rightarrow-\infty$. The initial position $\mathbf{x}$ is a parameter, in this equation, through the factor $\delta Y_{o} e^{-s_{q}}$, while $\bar{s}$ is a function only of $\tau$, dependent on the heat release. In terms of the new time variable

$$
\sigma=\delta \int_{-\infty}^{\tau} e^{-(\gamma-1)\left(\tau^{2}-\bar{s}\right)} d \tau
$$

the solution to Equation (24) is

$$
s=-\ln \left(1-\sigma / \sigma_{b}\right),
$$

which exhibits thermal runaway when $\sigma=\sigma_{b}$, where

$$
\sigma_{b}(\mathbf{x}) \equiv e^{s_{0}} / Y_{o} .
$$

Since Equation (26) gives $s$ explicitly as a function of $\sigma$ and $\sigma_{b}$ it may be used in Equation (22) to evaluate the average $\bar{s}$ as a function of $\sigma$, after which the function

$$
F(\sigma)=\int_{0}^{\sigma} e^{-(\gamma-1) s} d \sigma
$$

may be calculated. Since an integration of the differential of Equation (25) shows that, in terms of the error function $e r f$,

$$
F(\sigma)=\frac{\delta}{2}\left(\frac{\pi}{\gamma-1}\right)^{1 / 2}\left\{1+\operatorname{erf}\left[(\gamma-1)^{1 / 2} \tau\right]\right\},
$$

the known value of $\delta$ enables $F(\sigma)$ to be expressed explicitly in terms of the known stretched time variable $\tau$. 
This analysis ceases to apply once thermal runaway occurs because thereafter Equation (26) gives a meaningless result. If $\sigma_{b c}$ denotes the minimum value of $\sigma_{b}$ anywhere within the chamber, then from Equation (26) or (27) an ignition value of $\sigma$ is defined as $\sigma_{i}=\sigma_{b c}$. The thermal runaway event of ignition first occurs in the chamber at the stretched time $\tau=\tau_{i}$ at which $\sigma=\sigma_{i}$, determined by Equation (29). If $\bar{s}$ is negligibly small for $\sigma<\sigma_{i}$, then Equation (28) shows that

$$
F\left(\sigma_{i}\right)=\sigma_{i}
$$

This indeed is the usual situation; since $\tilde{s}$ is the volume average according to Equation (22), ignition would have to occur almost simultaneously throughout the entire volume for $\bar{s}$ not to be small, and in practice at most a few ignition points are observed, so that $\sigma_{b} \gg \sigma_{b c}$ in most of the chamber. From Equation (29), it therefore follows that the stretched ignition time may be calculated from

$$
1+\operatorname{erf}\left[(\gamma-1)^{1 / 2} \tau_{i}\right]=\left(2 \sigma_{b c} / \delta\right)[(\gamma-1) / \pi]^{1 / 2} .
$$

Equation (31) has no solution unless $\delta[\pi /(\gamma-1)]^{1 / 2}>\sigma_{b c}$, a critical condition that must be satisfied if ignition is to occur at all. If this condition is not satisfied, then cooling associated with the expansion following TDC prevents autoignition. If ignition is to occur prior to TDC, then it is necessary that $\tau_{i}<0$, and Equation (31) requires $\delta[\pi /(\gamma-1)]^{1 / 2}>2 \sigma_{b c}$. In the range of reactivities defined by $\sigma_{b c}<\delta[\pi /(\gamma-1)]^{1 / 2}<$ $2 \sigma_{b c}$, ignition occurs after TDC, the initial expansion being too weak to suppress the heating initiated during compression. In applications, to assure satisfaction of the criticality condition, with a good margin of safety, generally ignition is designed to occur before TDC.

The relationship of the present analysis to classical ignition theory is indicated in Appendix B.

\section{THE STAGE OF IGNITION-FRONT PROPAGATION}

The preceding results determine when ignition first occurs and can be used to give pressure histories over the ignition transient. Since Equations(18) and (21) show that $P=P_{c}\left[1+\gamma \varepsilon\left(\bar{s}-\tau^{2}\right)\right]$ during the ignition stage, the pressure increment over the inert pressure, $P_{c} \gamma \varepsilon \bar{s}$, is small during this period, because $\varepsilon$ and $\bar{s}$ are both small. Subsequent to that ignition stage is a stage of ignition-front propagation, during which ignition waves spread from the ignition points, and significant pressure rise occurs. During this stage, ignitions may also develop at other points that at time $t_{o}$ have values of their entropy parameter $S$ below $S_{c}$ by an amount of order larger than $\varepsilon$, but because of its greater heat release, the ignition propagation generally dominates the pressure rise, so that these subsequent ignitions may be neglected. The value of $\varepsilon$ is assumed here to be small enough that, after ignition occurs, combustion goes to completion in a negligibly short time across the ignition front, consuming all of the fuel or oxidizer, depending on which one is the deficient reactant. In $\mathrm{Cl}$ applications, cooling by processes such as fuel vaporization generally causes oxidizer-rich regions to be hotter, so that fuel initially is the deficient reactant in the ignition regions. Later the ignition fronts may propagate into fuel-rich regions, so that oxygen depletion controls the heat release. 
Multiplication of Equation (9) by $q /\left(c_{p} T\right)$, followed by addition to Equation (10), results in a source-free total-enthalpy equation that can be written as

$$
D S / D t+\left\{q /\left[c_{p} P^{(\gamma-1) / \gamma}\right]\right\} D Y_{F} / D t=0 .
$$

A fluid element at initial position $\mathbf{x}$ that burns at a time $t_{b}(\mathbf{x})$, at which time the pressure is $P_{b}(\mathbf{x})$, will experience an increase in $S$ upon burning by an amount $q X_{0} /\left[c_{p} P_{b}^{(\gamma-1) / \gamma}\right]$, according to the integral of Equation (32) across the short increment of time required for the element to burn, where $X$ is $Y_{F}$ for $Z<Z_{s}$ and $Y_{o} / v_{o}$ for $Z>Z_{s}$, the stoichiometric mixture fraction being $Z_{s}=Y_{O_{*}} /\left(v_{o}+Y_{o_{x}}\right)$. The function $X_{\boldsymbol{o}}(\mathbf{x})$ is a unique function of $Z_{o}(\mathbf{x})$. In the first approximation, $D S / D t$ vanishes both before and after burning, because the reaction rate is negligible in Equation(10) before burning, and $Y=0$ in Equation (10) after burning. Therefore, the solution for $S$ at leading order is

$$
S= \begin{cases}S_{o}(\mathbf{x}), & t<t_{b}(\mathbf{x}) \\ S_{o}(\mathbf{x})+q X_{o}(\mathbf{x}) /\left[c_{p} P_{b}(\mathbf{x})^{(\gamma-1) / / \gamma}\right], & \mathrm{t}>t_{b}(\mathbf{x}) .\end{cases}
$$

Use of Equation (33) in Equation (13) gives

$$
\left(\frac{P}{P_{c}}\right)^{1 / \gamma}=\frac{V_{0}}{V}\left\{1+\frac{1}{V_{a}} \iint_{V_{b}} \int\left[\frac{q X_{0} W_{o}}{c_{p} S_{o} W_{b} P_{b}^{(\gamma-1) / y}}+\frac{W_{o}}{W_{b}}-1\right] d V_{o}\right\}
$$

where $W_{b}(\mathbf{x})$ denotes the average molecular weight of the burnt gas, and $V_{b}(t)$ is the volume at $t=t_{o}$ of the fluid comprising the burnt gas at time $t$.

In the previous section, an expansion of Equation (13) was employed, near time $t_{\theta}$, in which fractional departures of $P$ and $V$ from $P_{c}$ and $V_{o}$ were of order $\varepsilon$. Once the fraction

$$
\alpha=V_{b}(t) / V_{o}
$$

of the initial fluid volume comprising burnt gas exceeds $\varepsilon$, terms of order $\varepsilon$ in the expansion of Equation (13) become negligible and can be omitted. In particular, the volume change does not contribute significantly to the pressure change during the short stage of ignition-front propagation. Since fractional pressure increases of order $\varepsilon$ are not of interest, henceforth all terms of order $\varepsilon$ will be ignored. In addition, for brevity the approximation $W_{b}=W_{o}$ will be employed, since changes in mean molecular weights for ignition-front propagaton usually are small, the mean molecular weight being dominated by nitrogen in most applications. Under these approximations, Equation (34) reduces to

$$
\left(P / P_{c}\right)^{1 / \gamma}=1+\alpha\langle G\rangle
$$

where \langle\rangle identifies the average over the $\mathbf{x}$ of the burnt gas alone, and

$$
G(\mathbf{x})=\left\{q X_{o}(\mathbf{x}) /\left[c_{p} T_{o}(\mathbf{x})\right]\right\}\left(P_{c} / P_{b}\right)^{(\gamma-1) / v},
$$

which is the fractional increase in temperature or $S$ upon burning. Equation (11) has been used in Equation (37) to write $S_{o} P_{c}^{(\gamma-1) / \gamma}$ as $T_{a}$, while $S_{o} P_{b}^{(\gamma-1) / \gamma}$ is the temperature of the fluid particle just prior to its ignition. Equation (36) is like an integral equation because it contains the volume integral of $G(\mathbf{x})$ which, according to Equation (37), involves the function $P_{b}(\mathbf{x})$, dependent on the evolution history and (contrary to the other functions) not known in advance. Nevertheless, Equation (36) indicates that 
finding the evolution of the burnt-gas volume fraction $\alpha$ is relevant to obtaining the pressure history during this stage.

The evolution of $\alpha$ is determined by the rate of propagation of the ignition front from each ignition point. The local ignition condition continues to be determined by $\sigma(\tau)=\sigma_{b}(\mathrm{x})$, where from Equations (17) and (27)

$$
\sigma_{b}(\mathbf{x})=e^{\left[S_{e}-S_{u}(\mathbf{x})\right] /\left(\varepsilon S_{c}\right)} / Y_{o}(\mathbf{x})
$$

At any given $\sigma$, the Lagrangian burnt-gas volume is simply that portion for which $\sigma_{b}(\mathbf{x})<\sigma$. Since the rate of propagation of surfaces at which $\sigma=\sigma_{b}$ thus determines the evolution of $\alpha$, it is most convenient to use $\sigma$ rather than $\tau$ as the working time variable for this stage; the relationship between $\tau$ and $\sigma$ can be addressed afterwards. An algorithm for calculating the rate of change of $\alpha$ with $\sigma$ can be stated by observing that associated with a change $d \sigma_{b}$ is a volume change $d V_{b}$, representing those volume elements in the chamber having values of $\sigma_{b}$ in the range $d \sigma_{b}$ about the value $\sigma_{b}$. These volume elements mainly will be thin layers surrounding burnt regions, but they may also include some newly ignited spots that previously had values of $\sigma_{b}$ greater than $\sigma$. The rate of increase of the burnt volume fraction is therefore determined by

$$
d \alpha / d \sigma=\left(d V_{b} / d \sigma\right) / V_{o}
$$

with $d V_{b} / d \sigma$ obtained from the inert calculation.

These results can be used for converting Equation (36) into an explicit expression for $P(\sigma)$. The increment $d \alpha$ in $\alpha$, associated with the change $d \sigma$ through Equation (39), provides an increment in pressure $d P_{b}(\mathbf{x})$ through Equation (36), the differential of which gives

$$
\gamma P^{(\gamma-1) / \gamma} d\left(P^{1 / \gamma}\right)=d P_{b}=\frac{P_{c}}{V_{o}} \iint_{d V_{b}} \int \frac{q X_{o}}{c_{v} T_{o}} d V_{o},
$$

where $c_{v}=c_{p} / \gamma$ is the specific heat at constant volume. Here use has been made of the fact that fluid elements in the volume element $d V_{b}$ have values of $P_{b}$ equal to the pressure $P$ at the time that they burn, so that the $P_{b}(\mathbf{x})$ in Equation (37) equals $P$ throughout $d V_{b}$ and can therefore be extracted from the integral and brought to the other side of the equation. The fractional contribution of a fluid parcel $d V_{o} / V_{o}$ to the pressure rise $d P_{b} / P_{c}$ is then seen from Equation (40) to be $q X_{o} /\left(c_{v} T_{o}\right)$, as would occur in constant-volume combustion. The integral of Equation (40) gives

$$
P / P_{c}=1+\alpha\left\langle q X_{o}(\mathbf{x}) /\left[c_{v} T_{o}(\mathbf{x})\right]\right\rangle
$$

as an expression for finding $P(\sigma)$ that is more useful than Equation (36) because the average multiplying $\alpha$ now can be obtained directly from the results of the inert calculation at time $t_{a}$.

The final step is to express the results in terms of $\tau$ (equivalent through Equation (18) to $t$ ) instead of $\sigma$. Equation (29) relates $\tau$ to $\sigma$, and to use this relationship, the integral $F(\sigma)$ must be evaluated in finding the stretched burning time $\tau(\sigma)$. From Equations (11), $(17),(18),(22),(33)$ and (37), in the integral of Equation (28) for obtaining $F(\sigma)$, the average stretched entropy variable is found to be

$$
\bar{s}=(\alpha / \varepsilon)\left\langle G S_{0} / S_{c}\right\rangle
$$


where contributions from regions outside the burnt volume have been neglected because they are small compared with those from regions inside. From Equations (25) or (28) and (42), with the small influences of the volume change neglected, the differential of Equation (29) is seen to give

$$
d \tau=e^{-(\gamma-1)(\alpha / \varepsilon)\left\langle G S_{o} / S_{\mathrm{c}}\right\rangle} \delta^{-1} d \sigma
$$

for $\tau>\tau_{i}$. Equation (43) can be integrated to find $\tau$ as a function of $\sigma$ subject to $\tau=\tau_{i}$ at $\sigma=\sigma_{i}=\sigma_{h e}$, after the exponential factor has been evaluated as a function of $\sigma$. If $\left(S_{o} / S_{c}\right)$ can be approximated as unity throughout the burnt-gas volume, which is true in the early part of this stage and is likely to fail only towards the end of ignition-front propagation when $X_{o}(\mathbf{x})$ is smaller in the parcels being burnt and consequent pressure rises are small, then use may be made of Equation (36) to write Equation (43) in the form

$$
d \tau=e^{\left.-(\gamma-1)\left(P_{b} / P_{c}\right)^{1 / \gamma}-1\right] / / z} \delta^{-1} d \sigma,
$$

which is simpler in that the only function involved is $P(\sigma)$, given by Equation (41).

Given $Z_{o}(\mathbf{x})$ and $S_{o}(\mathbf{x})$, the function $\sigma_{b}(\mathbf{x})$ can be obtained from Equation (38), because $Y_{o}(\mathbf{x})$ depends only on $Z_{o}(\mathbf{x})$, as indicated explicitly below. Next, $\alpha(\sigma)$ can be obtained from the integral of Equation (39), and then $P(\sigma)$ can be calculated from Equation (41), since $T_{0}(\mathbf{x})$ is known from $S_{0}(\mathbf{x})$ through Equation (11) and $X_{0}(\mathbf{x})$ from $Z_{o}(\mathbf{x})$ (see below). The integral of Equation (43) or of Equation (44) then enables $\alpha$ and $P$ to be expressed as functions of $\tau$. The histories are thereby readily computed when deterministic descriptions of the inert stage are available.

\section{IMPLICATIONS CONCERNING PROBABILISTIC DESCRIPTIONS}

The preceding considerations, indicating how results of inert-stage computations may be employed to determine the pressure evolution during the short stage of ignitionfront propagation, are phrased as if totally deterministic results were available for the inert stage. The turbulence modeling generally does not provide such results but instead deals with averages such as means and variances of flow variables. The modeling thus is fundamentally probabilistic, and it is relevant to consider how such probabilistic descriptions can be meshed with the results of the present paper. Because of the strong temperature sensitivity, it is wrong to apply the present ideas with inert-stage averages simply selected at $t=t_{o}$. Yet, it is impractical to ask turbulence modelers generate sets of deterministic realizations for use in conjunction with the present results. It is thus important to ask what kinds of probabilistic results are needed in correctly applying the methods developed here. Certain probability-density functions (PDF's) clearly are required, and it is desirable to state here precisely what these PDF's are. Knowing them, modelers may choose to try to develop ambitious PDFevolution techniques or simpler presumed-PDF methods for calculating them during the inert stage. Various methods are. reviewed by Liñan and Williams, (1993, pp. 140-149), for example. With the presumed-PDF approaches, moments available from suitable means and variances yield the PDF. 
To avoid creating realizations in probabilistic descriptions, at least the joint PDF for the mixture fraction $Z_{0}(\mathbf{x})$ and for the entropy-related variable $S_{0}(\mathbf{x})$ is needed from the inert calculations at time $t_{\sigma}$. For the ignition stage, no other information is required in obtaining a PDF for the ignition time from Equation (31), because the statistics arise there only through $\sigma_{b v}$, and $Y_{o}$ in Equation (27) or Equation (38) is uniquely related to $Z_{n}$, for example as

$$
Y_{o}=Y_{o_{x}}^{m}\left(1-Z_{0}\right)^{m} Z_{o}^{n}
$$

according to Equations (7) and (8) and the simplification indicated in the beginning of the formulation. For the stage of ignition-front propagation, the mass-fraction change $X_{0}$ also is needed, but since $X$ is related uniquely to $Z$, for example

$$
X_{0}= \begin{cases}Z_{o}, & Z_{o}<Z_{s} \\ \left(1-Z_{o}\right)\left(Y_{O_{2}} / v_{o}\right), & Z_{o}>Z_{s}\end{cases}
$$

under these same approximations, this need is already satisfied. From the PDF $\mathscr{P}\left(Z_{o}, S_{0} ; \mathbf{x}\right)$ at each position $\mathbf{x}$ in the chamber (the probability that the mixture fraction lies in the range $d Z_{v}$ about the value $Z_{o}$ and that the entropy-related variable lies in the range $d S_{o}$ about the value $S_{o}$ being $\mathscr{P} d Z_{0} d S_{0}$ ), standard probabilistic methods enable marginal PDF's for $\sigma_{b}$, Equation (38), and for $q X_{o} /\left(c_{v} T_{o}\right)$, appearing in Equation (41), to be generated from $\mathscr{P}$ at each $\mathbf{x}$. It is also necessary that, for a given $d \sigma_{b}$, a PDF for $d V_{b}$ must be obtainable for use in Equation (39) to generate a PDF for $d \alpha / d \sigma$. Finding the PDF for $d V_{b}$ subject to the given $d \sigma_{b}$ entails knowing the conditional PDF for $\left|\nabla \sigma_{b}\right|$, given $\sigma_{b}$, and since this must be known for all $\sigma_{b}$, its knowledge is equivalent to knowing the joint PDF $\mathscr{P}\left(\sigma_{b}\left|\nabla \sigma_{b}\right| ; \mathbf{x}\right)$. It therefore follows that, in general, the trivariate PDF $\mathscr{P}\left(Z_{o}, S_{o},\left|\nabla \sigma_{b}\right| ; \mathbf{x}\right)$ must be available. This is quite a large amount of statistical information.

Significant simplifications in these requirements arise from rather weak assumptions. For example, a very mild hypothesis of statistical independence enables knowledge of the two bivariate PDF's $\mathscr{P}\left(Z_{o}, S_{0} ; \mathbf{x}\right)$ and $\mathscr{P}\left(\sigma_{b},\left|\nabla \sigma_{b}\right| ; \mathbf{x}\right)$ to suffice. Although the last of these is analogous to $\mathscr{P}\left(Z_{o},\left|\nabla Z_{o}\right| ; \mathbf{x}\right)$, the well-known joint PDF for the mixture fraction and the "scalar dissipation" (Liñan and Williams, 1993, pp. 133-137), it pertains to a different variable, that determining ignition-front propagation, and it has not been identified previously as a bivariate PDF worthy of turbulence modeling. However, there are conditions under which knowledge of $\mathscr{P}\left(Z_{o},\left|\nabla Z_{o}\right| ; \mathbf{x}\right)$ is sufficient for all purposes. Namely, if $S_{o}$ is related uniquely to $Z_{o}$, then $\mathscr{P}\left(Z_{o}, S_{o} ; \mathbf{x}\right)$ contains a delta-function factor that enables ignition times to be obtained from the one-variable $\operatorname{PDF} \mathscr{P}\left(Z_{o} ; \mathbf{x}\right)$, and $\sigma_{b}$ also becomes uniquely dependent on $Z_{o}$, enabling $\mathscr{P}\left(\sigma_{b},\left|\nabla \sigma_{b}\right| ; \mathbf{x}\right)$ to be generated from $\mathscr{P}\left(Z_{o},\left|\nabla Z_{a}\right| ; \mathbf{x}\right)$.

Ignition and the predominant ignition-front propagation occur in the hot, oxygenrich portions of the gas. In these regions, diffusive effects are small, and on the average the influences of fuel vaporization during the inert stage can be taken into account through conservation relations that are just like Equations (1) and (3), except that the chemical source terms are replaced, respectively by $+\dot{m} / \rho$ and $-L \dot{m}$, where $\dot{m}$ is the local average mass of fuel vaporized per unit time per unit volume of space, and $L$ is the heat of vaporization per unit mass of the fuel. In the same way that Equation (10) is 
derived, it is then found that during the inert stage

$$
D S / D t=-\left[L /\left(c_{p} p^{(\gamma-1) / \gamma}\right)\right] D Y_{F} / D t .
$$

Since variations of $L / c_{p}$ and of $P$ typically are small during the ignition stage, they may be neglected here, so that $L / c_{p}$ may be treated as constant, and $P$ may be replaced by $P_{c}$. It then follows that $S+Y_{F} L /\left(c_{p} P_{c}^{(\gamma-1) / \gamma}\right)$ is constant, which enables the inert relationship $Y_{F_{o}}=Z_{o}$ to be used to derive the result

$$
S_{o}=S_{c}-Z_{o} L /\left(c_{p} P_{c}^{(\gamma-1) / \gamma}\right) .
$$

Equation (48) relates $S_{0}$ uniquely to $Z_{0}$ thereby reducing the problem to that of finding $\mathscr{P}\left(Z_{o},\left|\nabla Z_{o}\right| ; \mathbf{x}\right)$. For example, substitution of Equations (45) and (48) into Equation (38) provides a unique relationshp for $\sigma_{b}$ in terms of $Z_{v}$,

$$
\sigma_{b}(\mathbf{x})=e^{Z_{\mathrm{o}}(\mathbf{x}) L /\left(s c_{\mathrm{p}} T_{\mathrm{c}}\right)} /\left\{\left\{Y_{O_{x}}^{m}\left[1-Z_{o}(\mathbf{x})\right]^{m}\left[Z_{o}(\mathbf{x})\right]^{n}\right\},\right.
$$

which approaches infinity as $Z_{o}$ approaches 0 or 1 and which achieves a minimum value at an intermediate value of $Z_{o}$ that is quite small for small values of $\varepsilon$, because of the exponential factor. It is remarkable under these further, apparently reasonable approximations, only $\mathscr{P}\left(Z_{j} ; \mathbf{x}\right)$ is needed to obtain the statistics of the ignition time $t_{i}$, and only $\mathscr{P}\left(Z_{o},\left|\nabla Z_{o}\right| ; \mathbf{x}\right)$ is required for a probabilistic description of the pressure history $P(t)$ during the stage of ignition-front propagation.

\section{DISCUSSION}

The present study has focused on the ignition and inital propagation stages in CIengine combustion. The objective has been to eliminate the need for including finite-rate chemistry in the turbulence modeling by providing analytical descriptions of the processes influenced by reaction rates. The approach has been designed to make use of computational results for the inert compression, injection, atomization, vaporization and mixing processes. Procedures have been developed here for using these results to obtain the ignition time and the combustion-produced pressure increase during the initial pressure transient that follows ignition.

The formulation and analysis result in an expression for the ignition time, Equation (31), involving the minimum (at any point within the chamber volume) inert value at TDC, $\sigma_{b c}$, of the function $\sigma_{b}(\mathbf{x})$, given in Equation (27) or Equation (38), which depends only on the inert-stage entropy and mixture-fraction fields. This thermal-runaway function, the importance of which is demonstrated by Equation (26), encompasses the spatially variable part of the scaled overall chemical reaction rate in strongly temperature-dependent autoignition. Fluid parcels in the chamber with large values of $\sigma_{b}$ have relatively low entropy and low reaction rates, and therefore elements with smaller values of $\sigma_{b}$ ignite first. The Frank-Kamenetskii reaction-rate parameter $\delta$ of Equation (20), which involves the small reciprocal activation-energy parameter $\varepsilon$ of Equation (15), must be sufficiently large, compared with the minimum $\sigma_{b c}$ if ignition is to occur before TDC, or if it is to occur at all and not be prevented by cooling associated with the expansions after TDC. 
The inert calculations needed to obtain the thermal-runaway function $\sigma_{b}(\mathbf{x})$ usually must be extended past the actual ignition time by excluding ignition chemistry from the computation. In practice, the evolution of the functions $Z(\mathbf{x})$ (determining the $Y(\mathbf{x})$ in $\sigma_{b}$ ) and $S(\mathrm{x})$ during the ignition stage may not be negligible, and consideration might therefore be given to taking into account possible changes in the function

$$
\Sigma(\mathbf{x})=e^{\left[S_{c}-S(x)\right] /\left(\varepsilon S_{c}\right)} / Y(\mathbf{x})
$$

with time during this stage. For example, after the ignition time $t_{i}$ is determined, $\Sigma(\mathbf{x})$ may be evaluated at $t=t_{i}$, and its minimum value may be compared with $\sigma_{b i}$. If differences are appreciable, then $t_{0}$ may be changed from TDC to $t_{i}$, and a new estimate of $t_{i}$ may then be computed in the same manner as before. This procedure may be pursued iteratively until acceptable convergence is obtained. Other methods for including inert variations during the ignition stage might also be considered; the importance of addressing this question will remain uncertain until sample computations are performed. Because of the nearness of ignition to TDC in most applications and the turbulent fluctuations that may be expected to lead to nearly the same minima of $\Sigma(x)$ under these conditions, the influence of this portion of the inert evolution may be estimated to be small, even though the continuing injection does lead to gradual increase in inert pressure and mean inert temperature, through and slightly beyond TDC.

In chambers of practical shapes, spatial gridding problems are severe for both the inert calculations and the calculations of the stage of diffusion-controlled combustion. This is true quite generally, independently of the procedures proposed here. Smooth fits of $Z(\mathbf{x})$ and $S(\mathbf{x})$ through grid points are desired for applying the present approach in describing ignition and ignition-front propagation. Smooth interpolations between original grid points are sufficient; with the proposed procedure it is unnecessary to consider adaptive gridding around points where igniton has occurred, for example. At the time that the computations are switched from inert compression to diffusioncontrolled combustion, the grid can easily be taken to be the same in the two programs. The positions in the chamber through which the ignition front has propagated are readily identified from the function $\sigma_{b}(\mathbf{x})$. In the diffusion-controlled combustion, the underlying assumption is that everything that can burn has burnt, so that the injection, atomization, evaporation, mixing and heat release that occur are transport-controlled, with chemical equilibrium achieved at every point in the gas.

The rapid pressure rise associated with the ignition-front propagation is obtained from Equation (41), in terms of $\sigma_{b}(\mathbf{x})$ and the function $V_{b}\left(\sigma_{b}\right)$, the initial inert volume that has values of the thermal-runaway parameter less than or equal to $\sigma_{b}(\mathbf{x})$. The pressure rise produced by the combustion of any fluid element as the ignition front passes through it depends on its relative heat release, which in turn is a function of its mixture-fraction and entropy variables $Z(\mathbf{x})$ and $S(\mathbf{x})$. These variables also affect the proportionality between changes in $\sigma_{b}$ and in time, given by Equation (44), the integral of which then serves to relate the pressure rise to time by accounting for the strong increase with time of the average burnt-gas entropy during this combustion stage. In general, use of probabilistic inert-stage descriptions to generate probabilistic descriptions of the rapid pressure rise thus involves determining the trivariate PDF $\mathscr{P}\left(Z_{o}, S_{o},\left|\nabla \sigma_{b}\right| ; \mathbf{x}\right)$, but correlations quite likely are weak enough that the various simplifications described in the previous section will be quite sufficient. 
It has been seen in the previous section that, under plausible approximations, the stages of ignition and of ignition-front propagation can both be described from knowledge of the bivariate PDF $\mathscr{P}\left(Z_{o},\left|\nabla Z_{o}\right| ; \mathbf{x}\right)$. This is the same PDF that arises in optimal descriptions of diffusion-controlled combustion in reaction-sheet regimes of turbulent diffusion flames (Liñan and Williams, 1993, pp. 111-118, 133-137). That this $\mathrm{PDF}$ also is relevant to ignition-front propagation in $\mathrm{Cl}$ applications is an unanticipated result which arises from the approximate relationship between the thermalrunaway function $\sigma_{b}$ and the mixture fraction $Z_{b}$, shown in Equation (49). In the heated air containing small concentrations of fuel, where ignition and the main ignition-front propagation occur, the inert entropy depends mainly on the inert fuel concentration, which in turn depends on mix ture fraction, so that the combination of temperature and reactant concentration determining thermal runaway through $\sigma_{b}$ varies with $\mathbf{x}$ primarily only as a consequence of the mixture-fraction variation. The field of the mixture fraction thus dominates not only the diffusion-controlled combustion stage but also the ignition history that precedes this stage.

Note all of the chemical energy available is released during the rapid pressure rise because this process proceeds too rapidly for significant mixing to occur. A variable mixture-fraction field therefore remains in the chamber alter the pressure rise, with some fluid parcels having unburnt fuel and others unconsumed oxygen. The present results quite simply determine the total chemical energy released during the rapid pressure rise in terms of the inert distributions of $Z$ and $S$ just prior to that rise and thereby easily give the magnitude of this pressure rise. Specifically, in Equation (41) it is necessary only to put $\alpha=1$ and to take the average over the entire chamber volume to obtain the pressure rise. The chemical energy remaining after the pressure rise occurs is then released in diffusion-flame combustion that proceeds during the continuing injection, evaporation and mixing after the stage of ignition-front propagation. It is reasonabte to consider this later stage of heat release, during which most of the chemical energy is in fact liberated, to be diffusion-controlled, so that mixing rather than finite-rate chemistry determines the heat-release rate. The mixture fraction is an optimum variable to use in turbulence modeling of this diffusion-controlled combustion, as indicated above, and it serves to reduce the problem once again to one of inert mixing. The initial conditions for this problem are a vailable from the results at the end of the stage of ignition-front propagation by use of Equation (33) for $t>t_{b}(\mathbf{x})$ and the pressure at that time. Thus, when full use is made of the results developed in the present study, the entire history can be described by employing turbulence modeling only for the inert and diffusion-controlled stages, without introducing finite-rate chemistry into such modeling.

\section{CONCLUSIONS}

This analysis has provided a way to obtain the ignition time in $\mathrm{Cl}$ combustion and has indicated that, after ignition at one or several hot spots, there is a stage in which the chamber pressure increases rapidly with time as ignition fronts rapidly consume the deficient reactant, thus leading to local entropy and temperature increases. The results may be used in conjunction with computational approaches to remove chemical 
kinetics from the problem by providing the necessary link between the initial, inertmixing stage and the final, equilibrium nonpremixed-combustion stage.

\section{REFERENCES}

Aggarwal, S. K. (1989). Ignition Behaviour of a Multicomponent Fuel Spray, Combust. Flame, 76, 5.

Dold, J. W. (1985). Analysis of the Early Stage of Thermal Runaway, Quart. J. Mech. Appl. Math., 38, 361.

Dold, J. W. (1988). Analysis of Thermal Runaway in the Ignition Process, SIAM J. Appl. Math., 49, 459.

Heywood, J. B. (1988). Internal Combustion Engine Fundamentals, Ch. 10, McGraw-Hill, New York, 491.

IDEA (1993). Final Workshop, Imperial College, London, March 22-23, 1993.

Kassoy, D. R., Riley, N., Bebernes, J. and Bressan, A. (1989). The Confined Nondiffusive Thermal Explosion with Spatially Homogeneous Pressure Variation, Comb. Sci. Tech., 63, 45.

Kamimoto, T. and Kobayashi, H.(1991). Combustion Process in Diesel Engines, Prog. Energy Combust. Sci., 17,163 .

Liñán, A. and Williams, F. A. (1993). Fundamental Aspects of Combustion, Oxford University Press, New York.

Müller, U. C., Peters, N. and Liñan, A. (1992). Global Kinetics for n-Heptane Ignition at High Pressure, Twenty-Fourth Symposium (International) on Combustion, The Combustion Institute, Pittsburgh, 777.

Takagi, T., Fang, C. -Y., Kamimoto, T. and Okamoto, T. (1991). Numerical Simulation of Evaporation, Ignition and Combustion of Transient Sprays, Combust. Sci. Tech., 75, 1.

Zel'dovich, Ya. B. (1980). Regime Classification of an Exothermic Reaction with Nonuniform Initial Conditions, Combust. Flame, 39, 211.

\section{APPENDIX A: INFLUENCES OF DEPARTURES FROM ONE-STEP ARRHENIUS KINETICS}

As indicated in the introduction, the analysis in the present paper is predicated on a one-step, Arrhenius description of the ignition kinetics. Figure 2, based on results of Müller, Peters and Liñán (1992), augmented by some further idealizations shown as the solid curves, is included here for illustrative purposes, to show that there typically is a temperature range in which this approximation is poor. The low-temperature activation energy of $35 \mathrm{kcal} / \mathrm{mol}$, seen in Figure 2, compares favorably with some values of about $40 \mathrm{kcal} / \mathrm{mol}$, quoted for Diesel fuels (Heywood, 1988, p. 544); the complexities typically begin at somewhat higher temperatures. The purpose of the present appendix is only to indicate, in a very general fashion, simplified approaches that may be used to address the influences of these complexities in $\mathrm{Cl}$ applications. Only a discussion is given; the more detailed analysis is too long to be presented here.

The approximate four-step mechanism that captures the behavior shown in Figure 2 and that underlies the analyses by asymptotics for which results are plotted there (Müller, Peters and Liñán, 1992) has two low-temperature steps and two hightemperature steps, each with a reaction intermediate. In the temperature range of interest here (less than about $1100 \mathrm{~K}$ ), a steady-state approximation for the high- 


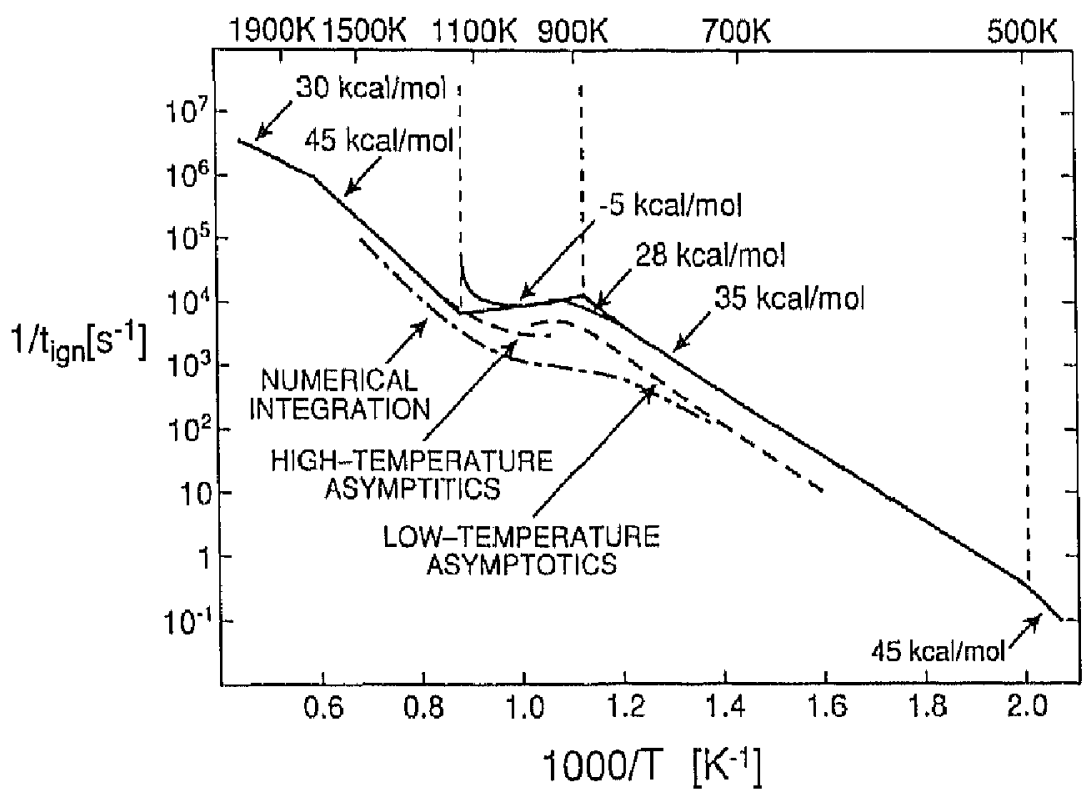

FIGURE 2 Homogeneous isobaric ignition times as functions of temperature for stoichiometric heptaneair mixtures at $40 \mathrm{~atm}$.

temperature intermediate is reasonable so that, with influences of reactant consumption during ignition neglected, the conservation equations for the mass fraction $Y_{I}$ of the low-temperature intermediate and for energy become

$$
D Y_{I} / D t=B_{1} P Y_{F} Y_{O} e^{-T_{1} / T}-B_{2} Y_{1} e^{-T_{2} / T}
$$

and

$$
\rho c_{p} D T / D t=d P / d t+\rho q\left(B_{3} P Y_{I} Y_{O} e^{-T_{3} / T}+B_{4} Y_{F} e^{-T_{1} / T}\right),
$$

which may be compared with Equations (1) and (3). Here $T_{1}=21,650 \mathrm{~K}, T_{2}=$ $37,285 \mathrm{~K}$ and $T_{3}=13,230 \mathrm{~K}$ for approximating heptane kinetics, and values of the prefactor constants (the $B$ 's) may be inferred from numbers in Müller, Peters and Liñán, (1992). The term involving $B_{4}$ in Equation (A2) arises from the high-temperature path, while the others pertain to the low-temperature path.

At the lowest temperatures, the reverse step involving $B_{2}$ is negligible, as is $B_{4}$, and a pair of equations is obtained, which may be treated in a manner paralleling the one-step AEA of the present paper but with greater complexity. At somewhat higher temperatures, the low-temperature intermediate achieves a steady state, so that from Equation (A1)

$$
Y_{I}=\left(B_{1} / B_{2}\right) P Y_{F} Y_{O} e^{\left(T_{2}-T_{2}\right) / T}
$$

which may be substituted into Equation (A2) to provide a one-step rate expression having two terms, one with the positive activation temperature $T_{1}$ and the other of activation temperature $T_{1}-T_{2}+T_{3}=-2405 \mathrm{~K}$, a negative value. The $B_{3}$ term involving this last activation temperature dominates in the region of negative temperature sensitivity in Figure 2, while the $B_{4}$ term involving $T_{1}$ dominates at higher temperatures. A model one-step rate that qualitatively accounts for the lowest- 
temperature range as well as reproducing these last results gives for energy conservation the form

$$
\rho c_{p} D T / D t=d P / d t+\rho q\left[A_{1} e^{-a T_{1} / T} /\left(1+A_{2} e^{-b T T_{1} / T}\right)+A_{3} e^{-T_{1} / T}\right],
$$

where the $A$ 's are constants involving the $B$ 's, $P$ and reactant mass fractions, and $a=0.8, b=0.9$. The rate in Equation (A4) is a smoothed version of the solid lines of Figure 2.

Let $R_{1}(T)$ and $R_{2}(T)$ denote the first and second term, respectively, in the brackets in Equation (A4), that is, the low-temperature rate and the high-temperature rate, and let $T_{a}$ and $T_{b}$ denote the temperatures at which the rate $R(T)=R_{1}(T)+R_{2}(T)$ is maximum and minimum, respectively $\left(T_{a}<T_{b}\right)$. There is another significant temperature $T_{d}<T_{a}$, such that $R\left(T_{d}\right)=R\left(T_{b}\right)$, that is, the rate at this lower temperature equals the rate at the minimum (which occurs at higher temperature). If compression ignition occurs below this lowest temperature, for example, if $T_{c} \leq T_{d}$ for the $T_{c}$ of the main text, then the low-temperature kinetics dominate ignition, since even the rate at the minimum exceeds that at ignition. If, on the other hand, compression ignition does not occur until the temperature exceeds $T_{b}$ (e.g., $T_{c} \geq T_{b}$ ), then the low-temperature path can be neglected, and the high-temperature kinetics dominate. Between these limiting regimes, that is, for compressional heating rates producing ignition temperatures between about $850 \mathrm{~K}$ and $1100 \mathrm{~K}$, the rate at the minimum, $R\left(T_{a}\right)$, is the slowest that the system encounters during the ignition process, and it is mainly this rate that then controls the ignition time, which becomes nearly independent of the ignition temperature in this temperature range, thereby requiring treatment beyond simple one-step AEA.

This intermediate temperature range defines the regime in which cool flames may be encountered in CI processes. If phenomena such as cool-flame propagation or slow CO oxidation in a second combustion stage are important, then the approach of the present paper does not completely remove the necessity of considering finite-rate chemistry in numerics. However, it seems likely that these complications will not arise in most applications (since ignition typically occurs below $850 \mathrm{~K}$ ) and that full use may therefore be made of the present results.

\section{APPENDIX B: RELATIONSHIP TO CLASSICAL IGNITION THEORY}

The results of Section 4 apply when compressional heating during the ignition transient enhances ignition. To illustrate further relationships to classical results, it may be helpful to observe that if compression becomes weak, then a different scaling is needed in Equation (18), such that the first equation is replaced by $\left(t-t_{1}\right) /\left(t_{i}-t_{1}\right)=\tau$, and the $\varepsilon \tau^{2}$ term is removed as being negligibly small. Here $t_{1}$ is the time at which the volume change became negligible, so that $V_{o}=V_{1}$ and, according to Equation (13), $P_{c}=P_{1}$, giving $S_{c}=S_{1}$ and $T_{c}=T_{1}$. The parameter $\delta$ is redefined by changing the exponent of $\varepsilon$ to -1 and replacing $t_{c}$ by $\left(t_{i}-t_{1}\right)$ in Equation (20). The $\tau^{2}$ term is absent in Equations (21), (24) and (25), and in Equation (25) the lower limit of the integral is now zero. The solution again is given by Equation (26), where now Equation (25) implies 
that $\delta \tau=F(\sigma)$. Since $\tau=1$ at ignition by the definition of $\tau$, Equation (29) is replaced by

$$
t_{i}-t_{1}=R^{0} c_{p} T_{c}^{2} e^{E / R^{n} T_{c}} F\left(\sigma_{c}\right) /\left(E q B_{c}\right) .
$$

In a homogeneous system, $s_{o}=0$ and $\bar{s}=s$, so that Equations (26) and (28) give

$$
F(\sigma)=\left[1-(1-\sigma Y)^{\gamma}\right] /(\gamma Y),
$$

and Equation (B1) becomes

$$
t_{i}-t_{1}=R^{0} c_{v} T_{\mathrm{c}}^{2} e^{E / R^{0} T_{c}} /\left(E q B_{c} Y\right),
$$

the Frank-Kamenetskii result for constant-volume ignition (see, for example, Liñán and Williams, 1993, p. 87). 